\title{
Clave dicotómica para especies parasitoides e hiperparasitoides (Hymenoptera) de áfidos (Hemiptera: Aphididae) de Costa Rica ${ }^{1}$
}

\section{Key to the species of parasitoids and hyperparasitoids (Hymenoptera) of aphids (Hemiptera: Aphididae) of Costa Rica}

\author{
Daniel Zamora-Mejías ${ }^{2}$, Paul E. Hanson ${ }^{3}$
}

\begin{abstract}
Resumen
Los áfidos representan una problemática para muchas especies de plantas, debido a que son vectores de múltiples patógenos; el control biológico mediante el uso de parasitoides es una opción permanente de control de sus poblaciones. El objetivo de esta investigación fue elaborar una clave dicotómica para identificar parasitoides e hiperparasitoides de áfidos recolectados en Costa Rica. Se obtuvo desde el año 2008 hasta 2015 un total de 3009 parasitoides himenópteros de 25 especies de áfidos, de los cuales 2832 (94\%) se clasificaron como parasitoides primarios y 175 (6\%) como hiperparasitoides. Se elaboró una clave de identificación para nueve especies de parasitoides primarios BraconidaeAphidiinae, Aphelinidae, Eulophidae y cinco familias de hiperparasitoides comprendidos en seis especies. Además, se incluye en la clave el género Quadrictonus (Aphidinae), el cual se registró por primera vez para Costa Rica en este estudio.
\end{abstract}

Palabras clave: control biológico, Aphidiinae, Aphelinidae, Encyrtidae, Eulophidae, Pteromalidae.

\begin{abstract}
Aphids represent a major problem for many plant species, due to their ability to serve as pathogen vectors; the use of parasitoids as biological control agents might be a suitable option for long term control of their populations. The objective of this study was to prepare a taxonomic key in order to identify parasitoids and hyperparasitoids of aphids obtained in Costa Rica. In total, during 2008 to 2015, 3009 hymenopteran parasitoids were reared from 25 species of aphids, 2832 (94\%) were primary parasitoids and $175(6 \%)$ were hyperparasitoids. A taxonomic key was made for the identification of nine species of primary parasitoids, Braconidae-Aphidiinae, Aphelinidae, Eulophidae, and six species of hyperparasitoids in five families. In addition, the genus Quadrictonus (Aphidiinae), was included in the key and was reported for the first time in this study in Costa Rica.
\end{abstract}

Keywords: biological control, Aphidiinae, Aphelinidae, Encyrtidae, Eulophidae, Pteromalidae.

\footnotetext{
Recibido: 9 de setiembre, 2016. Aceptado: 23 de febrero, 2017. Este trabajo fue elaborado parcialmente como parte de la tesis de Licenciatura en Biología, sustentada en 2009 por el primer autor, en la cual el segundo autor fungió como tutor en la Universidad de Costa Rica. Además incluye datos adicionales colectados como parte de las investigaciones independientes de los autores.

2 Universidad de Costa Rica, Sección de Biología. San Ramón, Alajuela, Costa Rica. A.P. 20201. josedaniel.zamora@ucr.ac.cr

3 Universidad de Costa Rica, Escuela de Biología. San Pedro de Montes de Oca, San José, Costa Rica. A.P.11501-2060.paul.hanson@ucr.ac.cr
} 


\section{Introducción}

Los áfidos representan una gran problemática agrícola por ser transmisores de patógenos, y pueden ser encontrados en una gran diversidad de plantas vasculares e incluso en briófitos que son usados como huéspedes (Blackman y Eastop, 1994; 2000; 2006). El conocimiento sobre la áfido-fauna costarricense y sus interacciones con las plantas huéspedes antes del año 2002, fueron escasas y solo se conocían pocas especies reportadas para el país, casi todas asociadas a cultivos agrícolas (Calvo, 1978; Chacón, 1980; Gómez, 1987; Hernández y Meneses, 1988; Meneses y Amador, 1988; Meneses et al., 1990). Sin embargo, desde la publicación de la primera lista de especies para Costa Rica (Voegtlin et al., 2003), el trabajo sobre este grupo se incrementó y se ha publicado investigación periódica sobre el tema (Pérez-Hidalgo et al., 2009; Quirós et al., 2009; Zamora-Mejías et al., 2010b; VillalobosMuller et al., 2010; Pérez-Hidalgo et al., 2012; Zamora-Mejías et. al. 2012; Pérez-Hidalgo y Villalobos, 2013).

Son pocos los ejemplos de especies de áfidos nativas de América Central (Nieto-Nafría et al., 2013; PérezHidalgo et al., 2012), ya que la mayoría son el resultado de introducciones por actividades humanas (Holman, 1974; Smith y Cermeli, 1979; Meneses et al., 1990; Étienne, 2005; Evans y Halbert, 2007; Quirós et al., 2009) o por invasión natural del hemisferio norte y sur (Villalobos-Muller et al., 2010), por ende, el ensamblaje costarricense de especies de áfidos refleja esta situación (Voegtlin et al., 2003; Pérez-Hidalgo et al., 2009; Quirós et al., 2009; Villalobos-Muller et al., 2010; Zamora-Mejías et al., 2010b; Pérez-Hidalgo et al., 2012).

Uno de los pasos importantes a seguir en el manejo de las poblaciones de áfidos es buscar medidas permanentes de control. El control biológico de áfidos utilizando enemigos naturales, especialmente de parasitoides bracónidos de la subfamilia Aphidiinae (Hymenoptera, Braconidae), los cuales parasitan exclusivamente áfidos, ha sido utilizado con éxito en otros países (Boivin et al., 2012), sin embargo, en Costa Rica no se ha implementado aún un programa de este tipo. Los afidiinos han sido utilizados extensamente en el control biológico, se conocen al menos nueve especies que han sido usadas para controlar las poblaciones de al menos catorce especies de áfidos (Boivin et al., 2012), por ejemplo Aphidius colemani y Lysiphlebus testaceipes han sido liberados con éxito para regular las poblaciones de Aphis gossypii (Soglia et al., 2006). Debido a lo anterior, su patrón de distribución en los trópicos americanos ha sido altamente influenciado por introducciones propias del control biológico, así como de manera accidental (Zamora-Mejías et al., 2010a; 2010b; 2011; 2012). Los afidiinos (subfamilia Aphidiinae) son endoparasitoides solitarios, koinobiontes, exclusivos de áfidos adultos e inmaduros (Hanson y Gauld 2006); su biología es bien conocida y atacan varias subfamilias de áfidos como Hormaphidinae, Lachninae, Greenideinae (Starý, 1970); sin embargo, en Costa Rica solamente se ha documentado su emergencia en especies de áfidos de la subfamilia Aphidinae (Zamora-Mejías et al., 2010a; 2011).

Existen limitantes para la implementación efectiva de control biológico, debido al manejo inadecuado de plagas mediante el uso de plaguicidas y por la presencia de hiperparasitoides, los cuales pueden limitar el efectivo control por parte de los parasitoides primarios (Rosenheim, 1998). El parasitismo secundario o hiperparasitismo es muy común en los áfidos, produciendo un efecto negativo en la dinámica poblacional de los parasitoides primarios (Brodeur y Rosenheim, 2000). Aun cuando solo parasitoides primarios sean introducidos, una gran y diversa comunidad de parasitoides secundarios se encuentran en los sistemas naturales como parte de la fauna nativa, por ejemplo Asaphes spp. (Pteromalidae) y Syrphophagus spp. (Encyrtidae) (Luck et al.,1981; Müller y Godfray, 1998).

El primer paso para implementar el control biológico de áfidos es la identificación de los parasitoides y en particular, la separación de los parasitoides primarios de los hiperparasitoides. La clave taxonómica es una herramienta para quienes trabajan con el control biológico de áfidos y quieren identificar las especies de parasitoides e hiperparasitoides presentes en determinada área geográfica. El objetivo de esta investigación fue elaborar una clave taxonómica dicotómica para identificar los parasitoides e hiperparasitoides conocidos de áfidos en Costa Rica. 


\section{Materiales y métodos}

Los parasitoides primarios y sus hiperparasitoides se obtuvieron al recolectar muestras de plantas infestadas de áfidos de diferentes áreas geográficas de Costa Rica dedicadas a diferente uso de suelo, incluyendo cultivos, ambientes urbanos, comunidades rurales y zonas protegidas (Zamora-Mejías et al., 2012), desde el año 2008 hasta 2015 (Pérez-Hidalgo et al., 2009; Zamora-Mejías et al., 2010a; Villalobos-Muller et al., 2010; Pérez-Hidalgo et al., 2012; Zamora-Mejías y Hanson, 2016), y especímenes recolectados en trampas Malaise $20 \mathrm{~km}$ sureste del Empalme (2800 msnm) y Villa Mills (3000 msnm), provincias de San José y Cartago respectivamente.

Para criar los parasitoides e hiperparasitoides de áfidos, se tomaron las partes de las plantas huésped con individuos momificados y no momificados con una colonia de áfidos de una única especie y se colocaron en cajas plásticas de $10 \mathrm{~cm}^{3}$, con un único orificio de ventilación de $5 \mathrm{~cm}^{2}$ cubierto con tela de algodón. Este método ayuda a evitar el rápido deterioro de las partes de las plantas, evitar la acumulación de humedad y la descomposición prematura de las muestras, hasta que emergieron los himenópteros.

Esta técnica también asegura la posibilidad de colectar áfidos adultos alados y no alados en etanol al 70\% para su identificación, mediante preparaciones microscópicas, utilizando el método de Bálsamo de Canadá, descrito con detalle en Voegtlin et al. (2003). La identificación de los especímenes fue realizada mediante las claves presentes en Blackman y Eastop (1994; 2000; 2006) y Voegtlin et al. (2003). En algunos casos se solicitó confirmación de las identificaciones a Nicolás Pérez Hidalgo (Universidad de León, España) y William Villalobos Müller (Universidad de Costa Rica).

Las avispas parasitoides e hiperarasitoides se conservaron en etanol al 70\%, y los Aphidiinae fueron identificados mediante claves taxonómicas para especies presentes en América (Starý, 1995; Wharton et al., 1998; Pike et al., 2000), y corroboradas por el especialista en este grupo taxonómico, Petr Starý (Instituto de Entomología, Academia de Ciencias de la República Checa). El género Quadrictonus (Braconidae: Aphidiinae) y los himenópteros no Aphidiinae fueron identificados utilizando las claves taxonómicas de los trabajos de Wharton et al. (1998), Gibson y Vikberg (1998) y Gibson (2001), y en el caso de Encyrtidae con la ayuda de John Noyes (Museo de Historia Natural, Londres). La clave dicotómica se elaboró mediante la revisión de las características morfológicas de los especímenes que son útiles para la separación de las especies. Las muestras de parasitoides primarios e hiperparasitoides se encuentran depositados en el Museo de Zoología de la Universidad de Costa Rica.

\section{Resultados y discusión}

En total, se procesaron 229 muestras de plantas que contenían colonias de áfidos, lo que resultó en 53 especies asociadas a 63 familias y 113 especies de plantas (lista completa en Zamora-Mejías et al., 2012). Sin embargo, solamente de 25 especies de áfidos (47\%) (Cuadro 1) se criaron parasitoides e hiperparasitoides. Emergieron un total de 3009 individuos, 94\% se clasificaron como parasitoides primarios y el 6\% como hiperparasitoides. Los especímenes criados incluían 2832 parasitoides primarios de la subfamilia Aphidiinae comprendidos en seis especies, un espécimen de Aphelinus (Aphelinidae) y otro de Tamarixia (Eulophidae), los cuales también son parasitoides primarios, y 175 especímenes de hiperparasitoides en cinco familias distribuidos en seis especies (Cuadro 2). El grupo de los parasitoides Aphidiinae estuvo dominado por dos especies, Lysiphlebus testaceipes (50\%) y Aphidius colemani (39\%), y las especies de hiperparasitoides criados de manera más frecuente durante esta investigación fueron Asaphes californicus (45\%), Pachyneuron aphidis (40\%) (ambos Pteromalidae) y Syrphophagus aphidivorus (13\%) (Encyrtidae).

En adición a los Aphidiinae criados de áfidos, hubo especímenes del género Quadrictonus recolectados en una trampa Malaise de tierras altas de Costa Rica. Hasta donde es posible determinar, este podría ser el primer registro 
Cuadro 1. Especies de áfidos de los que se criaron parasitoides primarios e hiperparasitoides en Costa Rica, mediante prospección de plantas hospederas y trampa Malaise, durante el periodo 2008-2015.

Table 1. Aphid species from which primary parasitoids and hyperparasitoids have been reared in Costa Rica, from aphid host plant samples and Malaise trap during the period time from 2008 to 2015.

\begin{tabular}{cc}
\hline Código huésped & Especie de áfido \\
\hline 1 & Aphis coreopsidis \\
2 & Aphis craccivora \\
3 & Aphis gossypii \\
4 & Aphis helianthi \\
5 & Aphis illinoisensis \\
6 & Aphis nerii \\
7 & Aphis spiraecola \\
8 & Aulacorthum solani \\
9 & Brachycaudus helichrysi \\
10 & Brevicoryne brassicae \\
11 & Hysteroneura setariae \\
12 & Illinoia morrisoni \\
13 & Lipaphis erysimi \\
14 & Lipaphis pseudobrassicae \\
15 & Macrosiphum salviae \\
16 & Microparsus pojanii \\
17 & Myzodium modestum \\
18 & Myzus ornatus \\
19 & Myzus persicae \\
20 & Pentalonia nigronervosa \\
21 & Rhopalosiphum maidis \\
22 & Sitobion avenae \\
23 & Toxoptera aurantii \\
24 & Toxoptera citricidus \\
25 & Uroleucon gravicorne \\
\hline
\end{tabular}

Cuadro 2. Parasitoides primarios* e hiperparasitoides** criados de áfidos en Costa Rica (código de huésped proviene del Cuadro 1) mediante cría de afidos en plantas huéspedes, durante el periodo 2008-2015.

Table 2. Primary parasitoids* and hyperparasitoids ** reared from aphids in Costa Rica (host codes from Table 1) from host plant samples during the period of time from 2008 to 2015.

\begin{tabular}{|c|c|c|c|}
\hline Superfamilia & Familia & Especie & Código huésped \\
\hline \multirow[t]{6}{*}{ Ichneumonoidea } & Braconidae & Aphidius colemani* & $2,3,6,9,18,19,20,21,24$ \\
\hline & & Binodoxys solitarius* & $3,8,9,13,18,19,23$ \\
\hline & & Diaeretiella rapae* $^{*}$ & $10,14,19$ \\
\hline & & Ephedrus lacertosus* & 25 \\
\hline & & Lipolexis oregmae* & 5,24 \\
\hline & & Lysiphlebus testaceipes* & $1,3,4,5,6,7,9,11,20,24$ \\
\hline \multirow[t]{6}{*}{ Chalcidoidea } & Aphelinidae & Aphelinus sp. * & Sin datos \\
\hline & Encyrtidae & Syrphophagus aphidivorus** & $3,6,24,25$ \\
\hline & Eulophidae & Tamarixia sp. * & 18 \\
\hline & Pteromalidae & Asaphes californicus ${ }^{* *}$ & $3,6,8,9,12,15,18$ \\
\hline & & Pachyneuron aphidis** & $3,6,8,20,24,25$ \\
\hline & Signiphoridae & Signiphora sp. ** & 5,24 \\
\hline Cynipoidea & Figitidae & Alloxysta sp. ** & 10 \\
\hline Ceraphronoidea & Megaspilidae & Dendrocerus sp. $* *$ & 22 \\
\hline
\end{tabular}


de este género fuera de México, donde fue criado a partir de un áfido de la tribu Macrosiphini (Wharton et al., 1998). Los especímenes de Costa Rica coinciden con las características presentadas en la clave para los géneros de Aphidiinae del Nuevo Mundo (Wharton et al., 1998).

Clave para la identificación de los parasitoides e hiperparasitoides de áfidos conocidos de Costa Rica (adaptada de Hanson y Gauld, 2006)

1 Ala anterior con al menos una celda cerrada o casi cerrada (Figuras 1a-1g). Antena filiforme, primer segmento de antena (escapo) no es el segmento más largo y no geniculada

Ala anterior sin celda, con una vena en el margen anterior, usualmente con una pequeña bifurcación cerca del ápice (Figuras 1h-2d). Antena geniculada, primer segmento de antena más largo que cualquier otro segmento. ........ 9

2 Ala anterior con una celda en forma semejante a un triángulo en el margen anterior (Figura 1a), sin otras celdas cerradas Alloxysta spp. (Figitidae)

Ala delantera sin una celda triangular (Figuras $1 \mathrm{~b}-1 \mathrm{~g}$ ) y a menudo con otras celdas cerradas. [Todos lo que siguen a partir de este paso son Braconidae-Aphidiinae.]

3 Ala anterior con 5-6 celdas cerradas (Figura 1b).

Ephedrus lacertosus

Ala anterior con menos de cinco celdas cerradas (Figuras 1c-1g).

4 Ala anterior con una celda grande y curva, cerrada en el ápice (Figuras 1c-1d) ............................................

Ala anterior sin celda grande y curva (Figuras 1e-1g).

5 Peciolo abdominal al menos tres veces más largo que ancho y en forma tubular con la parte dorsal y ventral fusionadas. Celda grande y curva del ala con dos venas en el ápice (Figura 1c) Quadrictonus sp. Peciolo mucho más corto y con dorso y vientre no fusionados. Celda grande y curva con una sola vena en el ápice (Figura 1d). Aphidius colemani

6 Parte basal del pecíolo abdominal en forma de un tubo, con dorso y vientre fusionados, la parte tubular alcanza al menos el nivel del espiráculo. Hembra con dos proyecciones alargadas y angostas en la parte posterior ventral del abdomen...... Binodoxys solitarius

Si hay una parte tubular en la base del pecíolo es muy corta y no alcanza el nivel del espiráculo. Hembra sin proyecciones en el abdomen.

7 Vena del ala anterior que se extiende desde la parte ventral del pterostigma se continúa con una vena parcial y transparente debajo (Figura 1e). Lysiphlebus testaceipes Vena que se extiende desde la parte ventral del pterostigma no se continúa con otra vena debajo (Figuras 1f$1 \mathrm{~g})$

8 Vena del ala anterior que se extiende desde la parte ventral del pterostigma es muy larga, alcanzando más de la mitad de la distancia hasta el ápice del ala (Figura 1f). Vainas del ovipositor curvadas hacia abajo.....Lipolexis oregmae Vena del ala anterior que se extiende desde la parte ventral del pterostigma es corta, no alcanzando la mitad de la distancia hasta el ápice del ala (Figura 1g). Vainas del ovipositor curvadas hacia arriba. Diaeretiella rapae

9 Margen anterior del ala anterior con pterostigma ensanchado, con vena estigmal larga pero sin vena postmarginal (Figura 1h). Mesoescudo con un surco en la parte media, en adición a los dos surcos laterales (notauli).

Dendrocerus sp. (Megaspilidae)

Ala anterior con pterostigma pequeña, vena estigmal usualmente más corta, vena postmarginal variable (Figuras 1i-2d). Mesoescudo usualmente sin surco en la parte media ......

10 Axilas casi tocándose en la porción media del escutelo (Figura 2a). Ala anterior con vena marginal muy corta

(Figura 2a). Tibia media con un espolón grande en el ápice. Syrphophagus aphidivorus (Encyrtidae) 
a

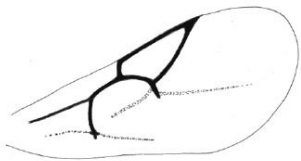

b

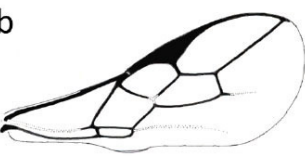

C

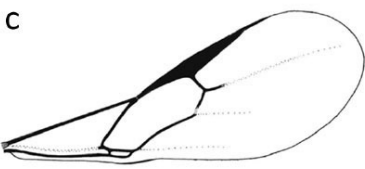

d

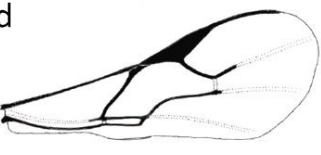

e

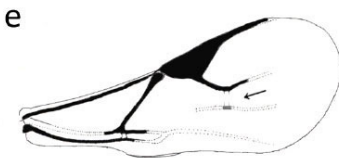

h

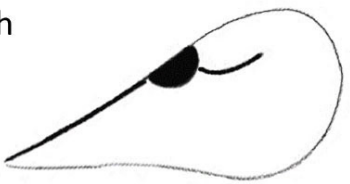

f
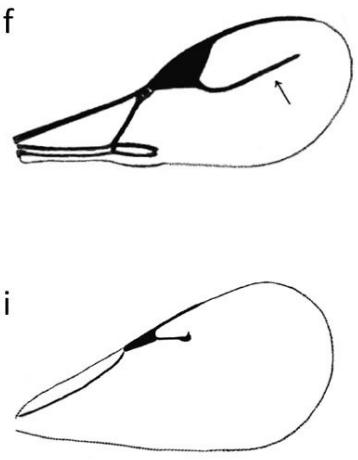

g
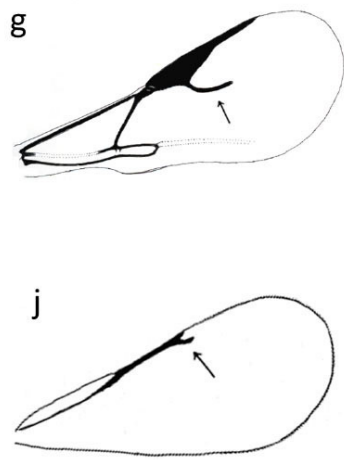

Figura 1. Ala delantera. a. Alloxysta sp. b. Ephedrus lacertosus. c. Quadrictonus sp. d. Aphidius colemani. e. Lysiphlebus testaceipes (la flecha indica la vena transparente). f. Lipolexis oregmae (la flecha indica la vena estigmal alargada). g. Diaeretiella rapae (la flecha indica la vena estigmal corta). h. Dendrocerus sp. i. Pachyneuron aphidis. j. Aphelinus sp. (la flecha indica la vena estigmal no muy evidente). (Figuras a y h-j modificadas de Hanson y Gauld (2006); Figuras b-e y g modificadas de Wharton et al. (1998); Figura f modificada de Rakhshani et al. (2012)).

Figure 1. Front wing. a. Alloxysta sp. b. Ephedrus lacertosus. c. Quadrictonus sp. d. Aphidius colemani. e. Lysiphlebus testaceipes (the arrow shows the transparent vein). f. Lipolexis oregmae (the arrow shows the long stigmal vein). g. Diaeretiella rapae (the arrow shows the short stigmal vein). h. Dendrocerus sp. i. Pachyneuron aphidis. j. Aphelinus sp. (the arrow shows the not very evident stigmal vein). (Figures a and h-j were modified from Hanson y Gauld (2006); Figures b-e and g were modified from Wharton et al. (1998); Figure f was modified from Rakhshani et al. (2012).

Axilas por lo general más separadas (Figuras 2b-2d). Vena marginal usualmente más larga (Figuras 1i-1j). Espolón apical de tibia media pequeño. 11

11 Clava antenal muy alargada, no dividida en segmentos (Figura 2b). Ala anterior con membrana casi libre de setas, pero con una hilera de setas largas en el borde.... Signiphora sp. (Signiphoridae)

Clava antenal no tan alargada (Figuras 2c-2d). Superficie de ala anterior normalmente con setas pequeñas. 12 12 Cuerpo usualmente con un brillo metálico (verde o azul). Antena con más de 10 segmentos........................ 13

Cuerpo sin brillo metálico. Antena con menos de 10 segmentos. 14

13 Mesoscudo con dos surcos (notauli) diagonales (Figura 2c). Vena marginal del ala anterior cuyo ancho es similar 
a

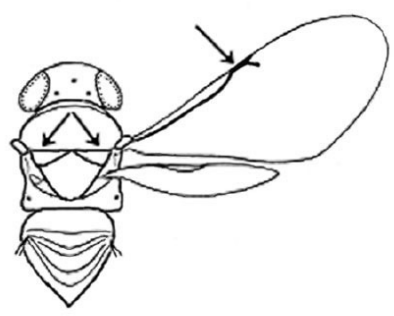

C

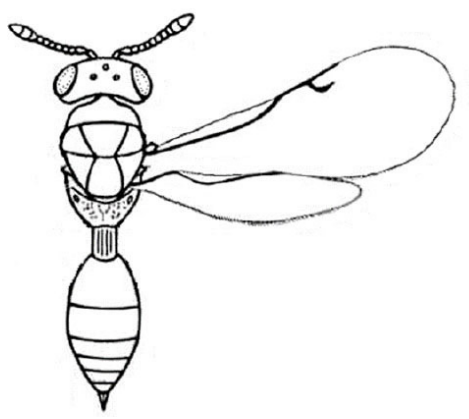

b
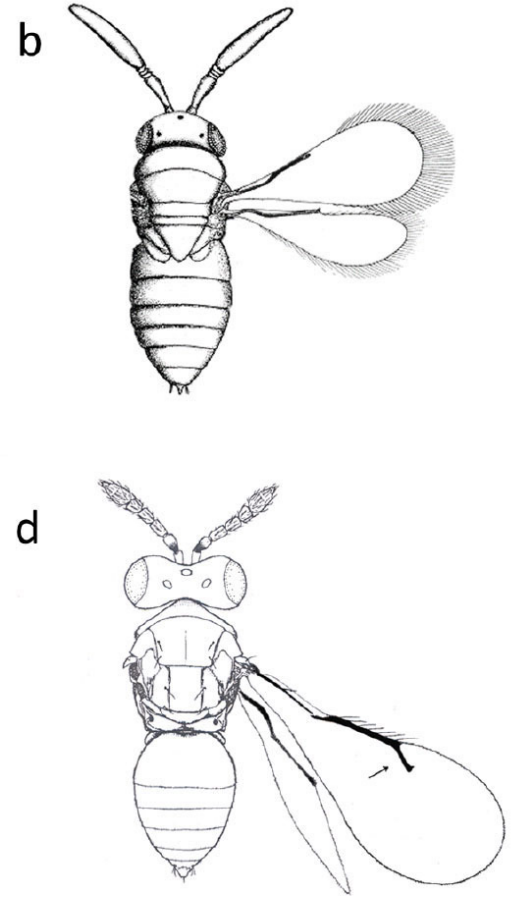

Figura 2. Cuerpo y alas en vista dorsal. a. Syrphophagus aphidivorus (las flechas indican las axilas casi tocándose y la vena marginal corta). b. Signiphora sp. c. Asaphes californicus. d. Tamarixia sp. (la flecha indica la vena estigmal muy evidente). (Figuras a-c modificadas de Hanson y Gauld (2006); Figura d modificada de Bouček (1988)).

Figures 2. Body and wings in a dorsal view. a. Syrphophagus aphidivorus (the arrows show the axillae nearly touching and the short marginal vein). b. Signiphora sp. c. Asaphes californicus. d. Tamarixia sp. (the arrow indicates the very evident stigmal vein). Figures a-c modified from Hanson and Gauld (2006); Figure d modified from Bouček (1988).

a las otras venas (Figura 2c). Pecíolo abdominal más largo que ancho (Figura 2c)

\section{Asaphes californicus (Pteromalidae)}

Mesoscudo sin surcos diagonales. Vena marginal a menudo más ancha que las otras venas (Figura 1i). Pecíolo más ancho que largo.

Pachyneuron aphidis (Pteromalidae)

14 Vena estigmal del ala anterior muy evidente (Figura 2d). Escutelo con dos líneas longitudinales paralelas (Figura 2d). Tamarixia sp. (Eulophidae)

15 Vena estigmal muy corta y no muy evidente (Figura 1j). Escutelo sin líneas longitudinales paralelas

Aphelinus sp. (Aphelinidae)

A pesar de las precauciones que se tomaron para evitar la cría de parasitoides que no se asocian a áfidos, es posible obtener ejemplares de la familia Mymaridae, a partir de huevos de insectos insertados en la vegetación (por ejemplo de Miridae y Cicadellidae). Los Mymaridae no son parasitoides de áfidos y son fáciles de identificar por la venación muy reducida en el ala anterior (generalmente hay una sola vena que no llega hasta la mitad de la longitud del ala), alas con muchas setas marginales (como en Signiphoridae; ver arriba) y antenas relativamente largas (Hanson y Gauld, 2006). 
En adición a las especies presentadas en el Cuadro 2 y en la clave, se obtuvieron dos morfoespecies de Aphidius no identificables a nivel de especie, debido a que varios de sus caracteres diagnósticos no se ajustaron a las descripciones taxonómicas presentes en la literatura (Zamora-Mejías et al., 2010a; 2010b). Sin embargo, se considera adecuado brindar una descripción breve en caso de que sean criados o colectados en algún momento por otros investigadores. Aphidius sp. A se caracteriza por la combinación de diecisiete segmentos antenales, índice tentorial 0,6 y palpos maxilares de cuatro segmentos. Aphidius sp. B criado en Uroleucon (Lambersius) gravicorne no cuenta con el número suficiente de especímenes para llegar a una conclusión definitiva, al igual que Binodoxys sp. criado en Aulacorthum solani del cual solamente se ha colectado un macho.

Durante esta misma investigación de Zamora-Mejías et al. (2010a; 2010b), se reportó la existencia de Aphidius sp. cercano a A. colemani, el cual está caracterizado por una combinación de 16-17 segmentos antenales (19 en machos), índice tentorial de 0,4 , cuatro palpos labiales, palpos maxilares tri-segmentados y las hembras con cuatro costas en el peciolo. Pertenece al grupo de especies de A. colemani y es posiblemente una especie distinta que aparece frecuentemente mezclada en la misma muestra, su estatus debe esperar reexaminación de los especímenes suramericanos de este grupo (Starý, comunicación personal, 2013). En Costa Rica se ha criado en Brachycaudus helichrysi, Illinoia morrisoni, Macrosiphum salviae y Myzodium modestum.

Entre los himenópteros no Aphidiinae presentados en el Cuadro 2, para cinco géneros no fue posible la identificación al nivel de especie (Aphelinus, Tamarixia, Signiphora, Alloxysta, Dendrocerus), debido a la escasez de especímenes criados (uno o dos especímenes cada uno) y, en la mayoría de los casos, la ausencia de claves para la identificación de las especies. La ocurrencia de Tamarixia asociadas a áfidos detectada en este trabajo es un dato interesante, ya que si bien existen al menos dos registros previos de parasitoides de este género sobre áfidos de otros países (Zuparko et al., 2011), la mayoría de las especies son parasitoides primarios de Psylloidea (Hemiptera), por lo cual, no está claro su papel en las interacciones tróficas con áfidos, y requiere futura investigación.

En el caso de Alloxysta (Figitidae), se conocen once especies de este género en Costa Rica, y existe una clave taxonómica para las especies (Ferrer-Suay et al., 2013); sin embargo, el registro presentado en esta investigación es el primer reporte de una especie de Alloxysta criado.

Los resultados aquí expuestos ponen en evidencia la necesidad de realizar más investigaciones en parasitoides de áfidos para Costa Rica, especialmente en tierras altas (altitudes mayores de $2000 \mathrm{msnm}$ ), donde es más probable la existencia de especies nativas de áfidos (Nieto-Nafría et al., 2013) y a la vez, se han registrado la mayoría de las especies de Alloxysta (Ferrer-Suay et al., 2013), y las del género Quadrictonus, un Aphidiinae (Braconidae) que aún no ha sido criado en el país.

\section{Agradecimientos}

A la Universidad de Costa Rica y a Idea Wild (USA) por el soporte logístico y económico brindado, a John Noyes (Museo de Historia Natural, Londres) por su ayuda con la identificación de Syrphophagus, a Nicolás Pérez Hidalgo (Departamento de Biodiversidad y Gestión Ambiental, Universidad de León España) y a William Villalobos-Müller (Centro de Investigación en Biología Celular y Molecular, Universidad de Costa Rica) por los especímenes brindados para completar este trabajo.

\section{Literatura citada}

Blackman, R.L., and V.F. Eastop. 1994. Aphids on the world's trees: An identification and information guide. CAB International, Oxon, GBR. 
Blackman, R.L., and V.F. Eastop. 2000. Aphids on the world's crops: An identification and information guid. $2^{\text {nd }}$ ed. John Willey \& Sons, Chichester, GBR.

Blackman, R.L., and V.F. Eastop. 2006. Aphids on the world's herbaceous plants and shrubs. Wiley, Chichester, GBR.

Boivin, G., T. Hance, and J. Brodeur. 2012. Aphid parasitoids in biological control. Canadian J. Plant Sci. 92:1-12.

Bouček, Z. 1988. Tamarixia leucaenae sp. n. (Hymenoptera: Eulphidae) parasitic on the lucaena psyllid Heteropsylla cubana Crawford (Hemiptera) in Trinidad. Bull. Ent. Res. 78:545-547.

Brodeur, J., and J.A. Rosenheim. 2000. Intraguild interactions in aphid parasitoids. Entomol. Exp. Appl. 97:93-108.

Calvo, C. 1978. Variación estacional del áfido Myzus persicae (Sulzer) en la Estación Experimental Agrícola Fabio Baudrit. Tesis MSc., Universidad de Costa Rica, San José, CRC.

Chacón, C. 1980. Evaluación de la población de áfidos alados en cultivos de papa para semilla en la zona norte de Cartago. Tesis MSc., Universidad de Costa Rica, San José, CRC.

Étienne, J. 2005. Les pucerons de Guadeloupe, des Grandes et Petites Antilles (Hemiptera, Aphididae). Bull. Soc. Entomol. France 110:455-462.

Evans, G.A., and S.E. Halbert. 2007. A checklist of the aphids of Honduras (Hemiptera: Aphididae). Fla. Entomol. 90:518-523.

Ferrer-Suay, M., J. Selfa, and J. Pujade-Villar. 2013. Review of the Neotropical Charipinae (Hymenoptera, Cynipoidea, Figitidae). Rev. Bras. Entomol. 57:279-299.

Gibson, A.P. 2001. The Australian species of Pachyneuron Walker (Hymenoptera: Chalcidoidea: Pteromalidae). J. Hymenoptera Res. 10:29-54.

Gibson, A.P., and V. Vikberg. 1998. The species of Asaphes Walker from America North of Mexico, with remarks on extralimital distributions and taxa (Hymenoptera: Chalcidoidea, Pteromalidae). J. Hymenoptera Res. 7:209-256.

Gómez, C. 1987. Fluctuación de la población de áfidos e incidencia de virus "Y” en tabaco en el cantón de Pérez Zeledón, provincia de San José, Costa Rica. Tesis MSc., Universidad de Costa Rica, San José, CRC.

Hanson, P.E., y I.D. Gauld. 2006. Hymenoptera de la Región Neotropical. American Entomological Institute, WI, USA.

Hernández, J., y R. Meneses. 1988. Nota descriptiva del pulgón lanígero (Erisoma lanigerum) de la manzana en Costa Rica. Manejo Integrado de Plagas 8:22-26.

Holman, J. 1974. Los áfidos de Cuba. Instituto Cubano del Libro, La Habana, CUB.

Luck, R., P.S. Messenger, and J.F. Barbieri. 1981. The influence of hyperparasitism on the performance of biological control agents. In: D. Rosen, editor, The role of hyperparasitism in biological control: a symposium. Division of Agricultural Sciences, University of California Publications, USA. p. 34-42.

Meneses, R., y R. Amador. 1988. Los áfidos alados de la papa y su fluctuación en Costa Rica. Manejo Integrado de Plagas 15:35-44.

Meneses, R., A. Ramírez, y G. Piaggio. 1990. Efectos de tres tipos de trampas de agua en la captura de áfidos. Manejo Integrado de Plagas 18:13-18.

Müller, C.B., and H.C. Godfray. 1998. The response of aphid secondary parasitoids to different patch densities of their host. BioControl 43:129-139.

Nieto-Nafría, J.M., N. Pérez-Hidalgo, D. Martínez-Torres, and W. Villalobos-Muller. 2013. A new aphid genus and species (Hemiptera: Aphididae: Macrosiphini) living on ferns in Costa Rica and Mexico. Can. Entomol. 145:509-520. 
Pérez-Hidalgo, N., D. Martínez-Torres, J.M. Collantes-Alegre, W. Villalobos-Muller, and J.M. Nieto-Nafría. 2012. A new species of Rhopalosiphum (Hemiptera, Aphididae) on Chusquea tomentosa (Poaceae, Bambusoideae) from Costa Rica. ZooKeys 166:59-73.

Pérez-Hidalgo, N., y W. Villalobos. 2013. Primera cita de la filoxera de la vid, Daktulosphaira vitifoliae (Fitch) (Hemiptera: Phylloxeridae) en Costa Rica. S.E.A. 53:335-336

Pérez-Hidalgo, N., W. Villalobos-Muller, and M.P. Mier-Durante. 2009. Greenidea psidii (Hemiptera: Aphididae: Greenideinae) new invasive aphid in Costa Rica. Fla. Entomol. 92:396-398.

Pike, K.S., P. Starý, T. Miller, G. Graf, D. Allison, L. Boydston, and R. Miller. 2000. Aphid parasitoids (Hymenoptera: Braconidae: Aphidiinae) of Northwest USA. Proc. Ent. Soc. Wash. 102:688-740.

Quirós, D.I., G. Remaudière, y J.M. Nieto-Nafría. 2009. Contribución al conocimiento de Aphididae y Phylloxeridae (Hemiptera, Sternorrhyncha) de Panamá. Neotrop. Entomol. 38:791-800.

Rakhshani, E., S. Kazemzadeh, P. Starý, H. Barahoei, N.G. Kavallieratos, A. Cetković, A. Popović, I. Bodlah, and Z. Toanović. 2012. Parasitoids (Hymenoptera: Braconidae: Aphidiinae) of northeastern Iran: Aphidiinae-aphid-plant associations, key and description of a new species. J. Insect Sci. 12:143.

Rosenheim, J.A. 1998 Higher-order predators and the regulation of insect herbivore populations. Ann. Rev. Entomol. 43:421447.

Smith, C.F., and M. Cermeli. 1979. An annotated list of Aphididae (Homoptera) of the Caribbean islands and South and Central America. N. C. Agr. Res. Serv. Tech. Bull. 259:1-131.

Soglia, M.C., V.H. Bueno, M.V. Sampaio, S.M. Rodrigues, and C.A. Ledo. 2006. Development and parasitism of Lysiphlebus testaceipes (Cresson) and Aphidius colemani Viereck (Hymenoptera: Braconidae) on Aphis gossypii Glover (Hemiptera: Aphididae) on two chrysanthemum cultivars. Neotrop. Entomol. 35:364-70.

Starý, P. 1970. Biology of aphid parasites (Hymenoptera: Aphidiidae) with respect to integrated control. Ser. Entomol. 6:1-643.

Starý, P. 1995. The Aphidiidae of Chile (Hymenoptera, Ichneumonoidea, Aphidiidae). Dtsch. Ent. Z. 42:113-138.

Villalobos-Muller, W., N. Pérez-Hidalgo, M.P. Mier-Durante, y J.M. Nieto-Nafría. 2010. Contribución al conocimiento de la fauna de pulgones (Hemiptera, Sternorrhyncha: Aphididae) de Costa Rica. Boln. Asoc. Esp. Ent. 34:145-182.

Voegtlin, D., W. Villalobos, M.V. Sánchez, G. Saborío, and C. Rivera. 2003. Guía de los áfidos alados de Costa Rica. Rev. Biol. Trop. 51: 1-228.

Wharton, R.A., P.M. Marsh, and M.J. Sharkey (eds.). 1998. Manual para los géneros de Braconidae (Hymenoptera) del Nuevo Mundo. International Society of Hymenopterists, WA, USA.

Zamora-Mejías, D., and P. Hanson. 2016. Trophic relationships of aphid hyperparasitoids (Hymenoptera) in Costa Rica. J. Entomol. Res. Soc. 18(3):35-44.

Zamora-Mejías, D., P.E. Hanson, and P. Starý. 2010a. Survey of the aphid parasitoids (Hymenoptera: Braconidae: Aphidiinae) of Costa Rica with information on their aphid (Hemiptera: Aphidoidea): plant associations. Psyche 2010:278643. doi: $10.1155 / 2010 / 278643$

Zamora-Mejías, D., P.E. Hanson, P. Starý, and E. Rakhshani. 2011. Parasitoid (Hym., Braconidae, Aphidiinae) complex of the black citrus aphid, Toxoptera citricidus (Kirkaldy) (Hem., Aphididae) in Costa Rica and its relationships to nearby areas. J. Entomol. Res. Soc. 13:107-115.

Zamora-Mejías, D., N. Pérez-Hidalgo, and M.P. Mier-Durante. 2010b. First report of Idiopterus nephrelepidis (Hemiptera: Aphididae) in Central America. Fla. Entomol. 93:460-463. 
Zamora-Mejías, D., N. Pérez-Hidalgo, W. Villalobos, and P. Hanson. 2012. New data about the Costa Rican aphid fauna (Hemiptera, Aphididae). Graellsia 68:305-312.

Zuparko, R.L., D.L. Queiroz, and J. LaSalle. 2011. Two new species of Tamarixia (Hymenoptera: Eulophidae) from Chile and Australia, established as biological control agents of invasive psyllids (Hemiptera: Calophyidae, Triozidae) in California. Zootaxa 2921:13-27. 